\title{
$S$-Parameter Formulation of Quality Factor for a Spiral Inductor in Generalized Two-Port Configuration
}

\author{
Tzyy-Sheng Horng, Member, IEEE, Kang-Chun Peng, Je-Kuan Jau, and Yu-Shun Tsai
}

\begin{abstract}
This paper formulates various quality $(Q)$ factors associated with the applications of on-chip spiral inductors to radiofrequency integrated circuits using $S$-parameters. The formulations start with the $Q$ factor of a spiral inductor in a generalized two-port configuration based on a new complex-power approach and then extend to the $Q$ factors of a tank and matching circuits that use the spiral inductors. In the demonstration, the two-port $S$-parameters for a series of CMOS spiral inductors have been measured to further generate such various $Q$ factors for a manysided evaluation of the inductor performance.
\end{abstract}

Index Terms-Complex power, on-chip inductor, quality $(Q)$ factor, $S$-parameters, spiral inductor.

\section{INTRODUCTION}

$\mathbf{S}$ PIRAL inductors are commonly used in radio-frequency integrated circuits (RFICs) to act as series or shunt elements in the tank, matching, and choke circuits. It is without controversy that the quality $(Q)$ factor is always the most important parameter to evaluate the inductor performance. However, to date, the spiral-inductor $Q$ factor has been formulated rigorously as the ratio of imaginary to real parts of the input impedance only in a one-port configuration with another port shorted [1]-[4]. This $Q$-factor formulation is good for spiral inductors when serving as shunt elements. Spiral inductors are also used frequently as series elements in many circuit applications and their performance will be distorted when evaluated using the $Q$ factor in one-port formulation. Several $Q$ factors have been calculated based on various two-port inductor models in the past [5]-[8]. It is common for them to ignore the shunt parasitic capacitances and resistances in the calculation of $Q$ factors. This will generally lead to overestimation of $Q$ factors below self-resonant frequency.

In our previous study [9], we considered the spiral inductor as a reciprocal two-port microwave network, as illustrated in Fig. 1. According to a general relation between its impedance network parameters and the complex power delivered to this network, we have successfully derived a closed-form expression for the $Q$ factor in terms of two-port $S$-parameters and load reflection coefficient. By setting minus unity for the load reflection

Manuscript received April 17, 2003; revised June 26, 2003. This work was supported by the R.O.C. Ministry of Education Program for Promoting Academic Excellence of Universities under Grant 91-E-FA08-1-4.

The authors are with the Department of Electrical Engineering, National Sun Yat-Sen University, Kaohsiung 804, Taiwan, R.O.C. (e-mail: jason@ ee.nsysu.edu.tw).

Digital Object Identifier 10.1109/TMTT.2003.818584

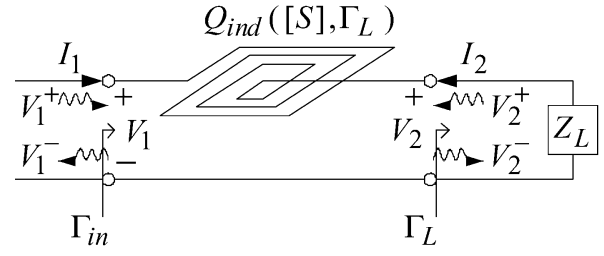

Fig. 1. Microwave network representation of a spiral inductor in a generalized two-port configuration.

coefficient to represent a short-circuit termination, the reduced expression of the $Q$ factor has been proven identical to that formulated in the one-port configuration. In this paper, we examine the spiral-inductor $Q$ factors more thoroughly by treating the load reflection coefficient as a variable parameter. This gives us another freedom to potentially improve $Q$ factors without the need to change the inductor geometrical or material parameters. The on-chip spiral inductors are commonly used with metal-insulator-metal (MIM) capacitors to form fundamental tank and matching circuits in RFICs. Typically, the inductor $Q$ factors are much lower than the capacitor $Q$ factors and will dominate the final $Q$ factors of circuits. Therefore, for simplicity, it can be acceptable to assume an infinite $Q$-factor quantity for the MIM capacitor in estimating the circuit $Q$ factor. By distinguishing the $Q$-factor definitions between spiral inductors and resonant circuits, we have found accurate conversion formulas to predict the $Q$ factors for tank and matching circuits from the spiral-inductor $Q$ factors in $S$-parameter formulation.

\section{FORMULATION}

In contrast to the $Q$ factor of a resonant circuit, the appropriate definition of the $Q$ factor for an inductor in referring to [1]-[3] is proportional to the net reactive energy stored, which is equal to the difference between the average magnetic and electric energies. Thus, the inductor $Q$ factor can be clearly expressed as

$$
Q_{\text {ind }}=2 \omega \frac{\left(W_{m}^{\mathrm{av}}-W_{e}^{\mathrm{av}}\right)}{P_{l}^{\mathrm{av}}}
$$

where $W_{m}^{\text {av }}, W_{e}^{\text {av }}$, and $P_{l}^{\text {av }}$ denote the average magnetic and electric energies stored and the average power dissipated in the inductor respectively. Fig. 1 shows a spiral inductor terminated by arbitrary load impedance $\left(Z_{L}\right)$. From Poynting's theorem applied in the frequency domain, the average complex power delivered to the input of the network can also be expressed in 
terms of the net reactive energy stored in and the average power dissipated by the network, i.e.,

$$
P_{\mathrm{av}}=P_{l}^{\mathrm{av}}+2 j \omega\left(W_{m}^{\mathrm{av}}-W_{e}^{\mathrm{av}}\right) .
$$

Comparing (1) and (2), one can find that the inductor $Q$ factor can be regarded as the ratio of the imaginary to the real part of the average complex power. From a circuit point-of-view, the average complex power can be rewritten as

$$
P_{\mathrm{av}}=\frac{1}{2}[V]^{t}[I]^{*}
$$

where the port voltage and current phasors are related to the incident and reflected voltage waves in the following forms:

$$
\begin{aligned}
{[V] } & =\left[V^{+}\right]+\left[V^{-}\right]=([U]+[S])\left[V^{+}\right] \\
{[I] } & =Z_{0}^{-1}\left(\left[V^{+}\right]-\left[V^{-}\right]\right)=Z_{0}^{-1}([U]-[S])\left[V^{+}\right] .
\end{aligned}
$$

Note that $[U]$ is an identity matrix. After substituting (4) and (5) into (3), one can expand $P_{\mathrm{av}}$ in terms of the $S$-parameters and incident voltage waves, and subsequently formulate the $Q$ factor as

$$
Q_{\text {ind }}=\frac{\operatorname{Im}\left\{P_{\mathrm{av}}\right\}}{\operatorname{Re}\left\{P_{\mathrm{av}}\right\}}=\frac{\left[V^{+}\right]^{t}\left\{j^{-1}\left([S]^{t}-[S]^{*}\right)\right\}\left[V^{+}\right]^{*}}{\left[V^{+}\right]^{t}\left([U]-[S]^{t}[S]^{*}\right)\left[V^{+}\right]^{*}} .
$$

As a matter of fact, the $Q$-factor expression in (6) is generally good for an arbitrary multiport inductive component. As derived in [9], the $Q$ factor for a spiral inductor when viewed as a reciprocal two-port component can be formulated as function of two-port $S$-parameters and load reflection coefficient. The resulting expressions are shown in (7) and (8) at the bottom of this page. In [9], we have also proven that the conventional $Q$ factor expressed as the ratio of the real to imaginary part of input impedance was the special case of (7) under the condition of short-circuit termination, i.e.,

$$
Q_{\text {ind }}\left([S], \Gamma_{L}=-1\right)=\frac{\operatorname{Im}\left\{\frac{\left(1+\Gamma_{\text {in }}\right)}{\left(1-\Gamma_{\text {in }}\right)}\right\}}{\operatorname{Re}\left\{\frac{\left(1+\Gamma_{\text {in }}\right)}{\left(1-\Gamma_{\text {in }}\right)}\right\}}=\frac{\operatorname{Im}\left\{Z_{\text {in }}\right\}}{\operatorname{Re}\left\{Z_{\text {in }}\right\}}
$$

where

$$
\Gamma_{\text {in }}=\frac{V_{1}^{-}}{V_{1}^{+}}=S_{11}+S_{12} F=S_{11}+\frac{S_{12} S_{21} \Gamma_{L}}{1-S_{22} \Gamma_{L}} .
$$

For demonstration, five on-chip spiral inductors fabricated in a $0.25-\mu \mathrm{m}$ RF CMOS process have been measured with the

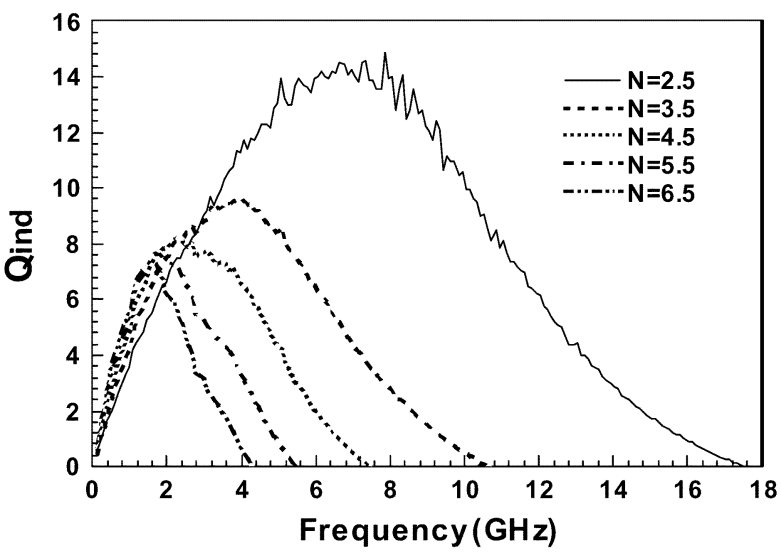

Fig. 2. Calculated $Q$ factors under short-circuit termination for CMOS spiral inductors with different turns.

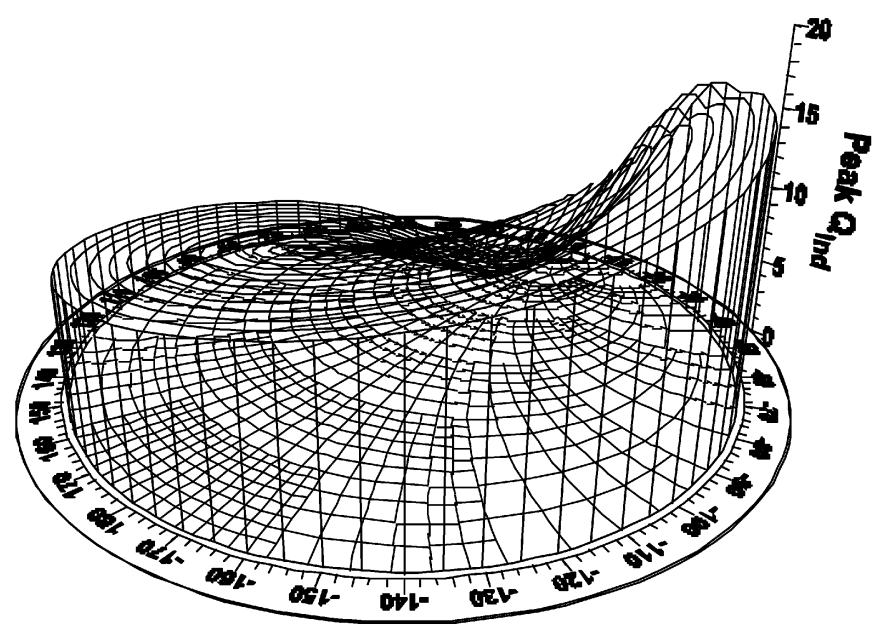

Fig. 3. Three-dimensional plot over a Smith chart for illustrating the peak $Q$ factor of a 3.5-turn CMOS spiral inductor as function of load reflection coefficient.

two-port $S$-parameters. Their layout parameters include $10-\mu \mathrm{m}$ -width, 2- $\mu \mathrm{m}$ spacing, $120-\mu \mathrm{m}$ inner dimension, and the number of turns $(N)$ from 2.5 to 6.5 . The inductances extracted at low frequencies for $N=2.5,3.5,4.5,5.5$, and 6.5 correspond to $2.2,3.7,5.9,8.6$, and $12.1 \mathrm{nH}$, respectively. The $Q$ factors calculated using (9) are shown in Fig. 2. It can be seen that the peak values of $Q$ factors range from 14.9 to 7.4, which basically decreases as the number of turns increases. Fig. 3 shows a three-dimensional plot over a Smith chart to illustrate the peak $Q$ factor of a 3.5-turn spiral inductor as function of load reflection coefficient according to (7). We have seen that, if the phase of the load reflection coefficient is fixed, the peak $Q$ factor increases as the magnitude of the load reflection coefficient increases. On

$$
\begin{aligned}
Q_{\text {ind }}\left([S], \Gamma_{L}\right) & =\frac{2 \operatorname{Im}\left\{S_{11}+S_{22}|F|^{2}\right\}+4 \operatorname{Im}\left\{S_{21}\right\} \operatorname{Re}\{F\}}{\left(1-\left|S_{11}\right|^{2}-\left|S_{21}\right|^{2}\right)+\left(1-\left|S_{22}\right|^{2}-\left|S_{12}\right|^{2}\right)|F|^{2}-2 \operatorname{Re}\left\{\left(S_{11} S_{12}^{*}+S_{21} S_{22}^{*}\right) F^{*}\right\}} \\
F & =\frac{S_{21} \Gamma_{L}}{1-S_{22} \Gamma_{L}}
\end{aligned}
$$



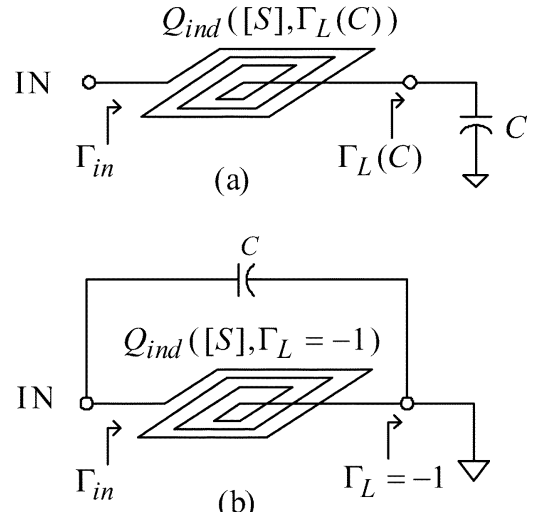

(b)

Fig. 4. $L C$ tank circuits using spiral inductors. (a) Series $L C$ tank. (b) Parallel $L C$ tank.

the other hand, if the magnitude is fixed instead, the major trend of the peak $Q$ factor with respect to the phase is to increase at the transition from an inductive load to a capacitive load, but to decrease at the transition from a capacitive load to an inductive load. We have searched for the maximum value of peak $Q$ factors over all possible load reflection coefficient points on a Smith chart. The resulting quantity is larger by $75 \%$ than that calculated at the short-circuit point.

\section{APPLICATIONS}

\section{A. LC Tank Circuits Using Spiral Inductors}

As mentioned before, the MIM capacitors in a normal semiconductor process usually have much higher $Q$ factors than the spiral inductors. When both components are connected to form series or parallel tank circuits, the spiral-inductor $Q$ factor is always considered as the dominant factor. Therefore, the capacitor can be treated as lossless for simplicity in analysis. For the series tank circuit shown in Fig. 4(a), the load reflection coefficient is a function of capacitance and can be expressed as

$$
\Gamma_{L}(C)=e^{-j \theta(\omega)}
$$

where

$$
\theta(\omega)=2 \tan ^{-1}\left(\omega C Z_{0}\right)
$$

At the angular resonant frequency $\left(\omega_{r}\right)$, the imaginary part of the input reflection coefficient is equal to zero. Thus,

$$
\begin{aligned}
\operatorname{Im}\left\{\Gamma_{\text {in }}\right\} & =\operatorname{Im}\left\{S_{11}+\frac{S_{12} S_{21} \Gamma_{L}}{1-S_{22} \Gamma_{L}}\right\} \\
& =\operatorname{Im}\left\{S_{11}+\frac{S_{12} S_{21} e^{-j \theta\left(\omega_{r}\right)}}{1-S_{22} e^{-j \theta\left(\omega_{r}\right)}}\right\} \\
& =0 .
\end{aligned}
$$

One can solve for $\theta\left(\omega_{r}\right)$ from (13) analytically and substitute it into (11) to find the load reflection coefficient and, subsequently, the spiral-inductor $Q$ factor using (7). For the case of a parallel tank circuit, as shown in Fig. 4(b), it is much simpler to set the load reflection coefficient equal to minus unity directly.

The definition in (1) shows that the $Q$ factor in an inductor is proportional to the net reactive energy stored, which is equal to

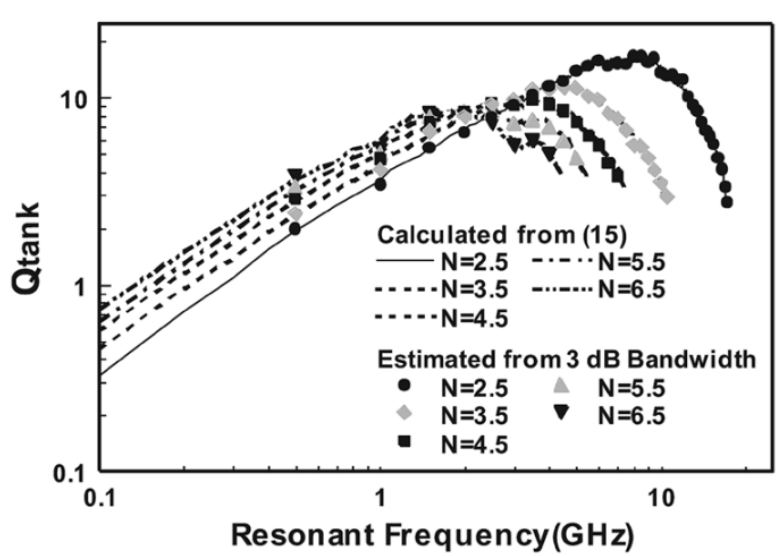

Fig. 5. Calculated $Q$ factors for series $L C$ tank circuits that use CMOS spiral inductors.

the difference between the average magnetic and electric energies. Due to some extra parasitic capacitances, a spiral inductor has a self-resonance at the angular frequency $\omega_{0}$ when the average electric and magnetic energies are equal. Therefore, its $Q$ factor will vanish to zero at $\omega_{0}$. For a tank circuit, its $Q$ factor is proportional to the average magnetic or electric energy stored at $\omega_{r}$. Therefore, the ratio between two $Q$ factors at $\omega_{r}$ is given as

$$
\frac{Q_{\mathrm{tank}}}{\left.Q_{\mathrm{ind}}\right|_{\omega_{r}}}=\frac{\left.W_{m}^{\mathrm{av}}\right|_{\omega_{r}}}{\left.W_{m}^{\mathrm{av}}\right|_{\omega_{r}}-\left.W_{e}^{\mathrm{av}}\right|_{\omega_{r}}}=\frac{1}{1-\frac{\left.W_{e}^{\mathrm{av}}\right|_{\omega_{r}}}{\left.W_{m}^{\mathrm{av}}\right|_{\omega_{r}}}} .
$$

One should caution that $W_{e}^{\text {av }}$ in (14) represents the average electric energy stored in the spiral inductor due to the existent parasitic capacitance. If only fundamental effects are considered, an equivalent simple parallel $R L C$ circuit can represent such a spiral inductor at self-resonance. For fixed magnitude of the input voltage, this equivalent parallel $R L C$ circuit exhibits that the average magnetic energy stored is proportional to $1 / \omega^{2}$, while the average electric energy stored is constant with frequency. With another fact that the average magnetic and electric energies stored in a spiral inductor are equal at $\omega_{0},(14)$ can be further approximated as

$$
\frac{Q_{\mathrm{tank}}}{\left.Q_{\mathrm{ind}}\right|_{\omega_{r}}} \approx \frac{1}{1-\frac{\left.W_{e}^{\mathrm{av}}\right|_{\omega_{0}}}{\left.W_{m}^{\mathrm{av}}\right|_{\omega_{r}}}}=\frac{1}{1-\frac{\left.W_{m}^{\mathrm{av}}\right|_{\omega_{0}}}{\left.W_{m}^{\mathrm{av}}\right|_{\omega_{r}}}} \approx \frac{1}{1-\left(\frac{\omega_{r}}{\omega_{0}}\right)^{2}} .
$$

According to (15), knowledge of the $Q$ factor of the spiral inductor and its self-resonant frequency can further estimate the $Q$ factor of any series or parallel tank circuit that uses this spiral inductor. Figs. 5 and 6 show the calculated results of $Q$ factors for series and parallel tank circuits shown in Fig. 4(a) and (b), respectively. These figures are plotted in the log-log scale to clearly show an increase rate approximately $10 \times$ per decade of resonant frequency for the tank-circuit $Q$ factors before the peak values. In addition, the spiral inductor with a larger inductance or number of turns corresponds to a larger $Q$ factor within this range. However, the spiral inductor with a smaller inductance or number of turns can achieve a higher peak value at a higher resonant frequency. Another estimation of $Q$ factors for 


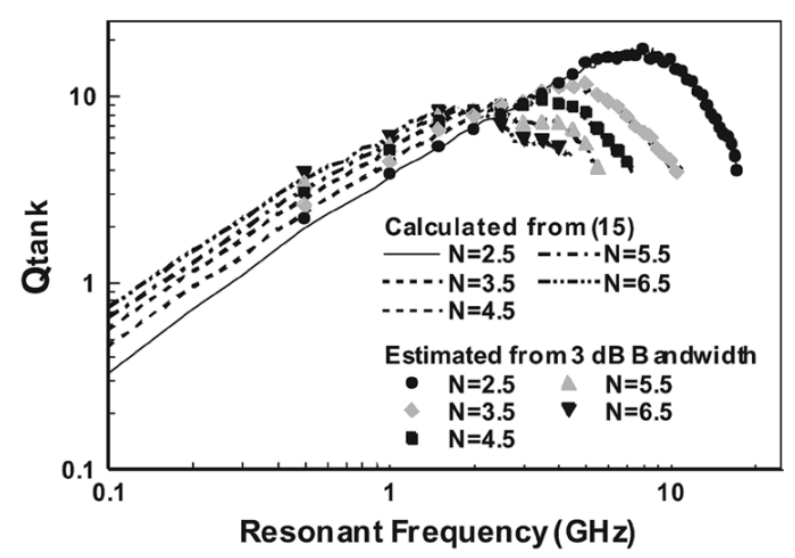

Fig. 6. Calculated $Q$ factors for parallel $L C$ tank circuits that use CMOS spiral inductors.

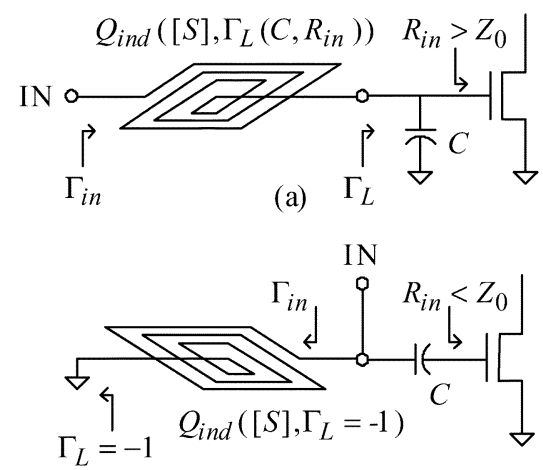

(b)

Fig. 7. L-section matching circuits using spiral inductors. (a) Matching circuit for high load impedance. (b) Matching circuit for low load impedance.

tank circuits can be done from the inverse of $-3-\mathrm{dB}$ fractional bandwidth of the input impedance or admittance responses [2], [3]. The results are also demonstrated in Figs. 5 and 6 for comparison. Excellent agreement can be found.

\section{B. L-Section Matching Circuits Using Spiral Inductors}

L-section networks shown in Fig. 7 are the simplest type of matching circuits for RFIC devices. There are two fundamental configurations for the networks matched to high and low load impedances, as shown in Fig. 7(a) and (b), respectively. A series spiral inductor is mainly used in the high-impedance-type matching circuit shown in Fig. 7(a). The reflection coefficient seen looking toward the input of the inductor has to be zero at the angular matching frequency $\left(\omega_{m}\right)$, i.e.,

$$
\Gamma_{\text {in }}=S_{11}+\frac{S_{12} S_{21} \Gamma_{L}}{1-S_{22} \Gamma_{L}}=0, \quad \text { at } \omega=\omega_{m}
$$

From (16), we can solve for $\Gamma_{L}$ in terms of $S$-parameters and substitute it into (7) to find the $Q$ factor of spiral inductors under the matching condition. At the matching frequency, the matching circuit acts as a resonant circuit and its unload $Q$ factor can be found from (15) as

$$
Q_{\text {tank }} \approx \frac{Q_{\text {ind }}\left([S], \Gamma_{L}=\frac{S_{11}}{\left(S_{11} S_{22}-S_{12} S_{21}\right)}\right)}{1-\left(\frac{\omega_{m}}{\omega_{0}}\right)^{2}} .
$$

The external $Q$ factor of the matching circuit that accounts for the loading effects can apply the well-known formula [10] given as

$$
Q_{e}=\sqrt{\frac{R_{\text {in }}}{Z_{0}}-1}
$$

where $R_{\text {in }}$ denotes the load resistance and has to satisfy the following matching condition:

$$
\begin{aligned}
R_{\text {in }}^{-1} & =Z_{0}^{-1} \operatorname{Re}\left\{\frac{1-\Gamma_{L}}{1+\Gamma_{L}}\right\} \\
& =Z_{0}^{-1} \operatorname{Re}\left\{\frac{S_{11}\left(S_{22}-1\right)-S_{12} S_{21}}{S_{11}\left(S_{22}+1\right)-S_{12} S_{21}}\right\} .
\end{aligned}
$$

For the low-impedance-type matching circuit shown in Fig. 7(b), a shunt spiral inductor is used instead and its $Q$ factor can be found simply by substituting $\Gamma_{L}=-1$ into (7). The corresponding unloaded $Q$ factor and external $Q$ factor [10] for this matching circuit can be found as

$$
\begin{aligned}
Q_{\mathrm{tank}} & \approx \frac{Q_{\text {ind }}\left([S], \Gamma_{L}=-1\right)}{1-\left(\frac{\omega_{m}}{\omega_{0}}\right)^{2}} \\
Q_{e} & =\sqrt{\frac{Z_{0}}{R_{\text {in }}}-1}
\end{aligned}
$$

where $R_{\text {in }}$ also has to satisfy the following matching condition:

$$
\begin{aligned}
R_{\text {in }} & =Z_{0} \operatorname{Re}\left\{\frac{1+\Gamma_{\text {in }}}{2 \Gamma_{\text {in }}}\right\} \\
& =Z_{0} \operatorname{Re}\left\{\frac{\left(1+S_{11}\right)\left(1+S_{22}\right)-S_{12} S_{21}}{2 S_{11}\left(1+S_{22}\right)-2 S_{12} S_{21}}\right\} .
\end{aligned}
$$

The loaded $Q$ factors $\left(Q_{\text {match }}\right)$ for the matching circuits can be finally evaluated using

$$
\frac{1}{Q_{\text {match }}}=\frac{1}{Q_{\operatorname{tank}}}+\frac{1}{Q_{e}} .
$$

As a result, Figs. 8 and 9 show the calculated $Q$ factors for the matching circuits shown in Fig. 7(a) and (b), respectively. It can be seen that each spiral inductor is successful in serving as a matching element in a limited range of matching frequency. Within this range, the increase rate of the $Q$ factor for a highimpedance-type matching circuit approaches $10 \times$ per decade of matching frequency. In addition, the spiral inductor with a larger inductance or number of turns corresponds to a higher $Q$ factor. On the contrary, the low-impedance-type matching circuit has a $Q$ factor approximately $10 \times$ smaller per decade of matching frequency in the applicable range. The spiral inductor with a larger inductance or number of turns corresponds to a lower $Q$ factor. From the external $Q$ factor $\left(Q_{\mathrm{e}}\right)$ expressions shown in (18) and (21), one can understand that, for a lossless matching circuit, a higher $Q$ factor implies a higher impedance transform ratio. However, from (23), the actual $Q$ factor $\left(Q_{\text {match }}\right)$ of a 


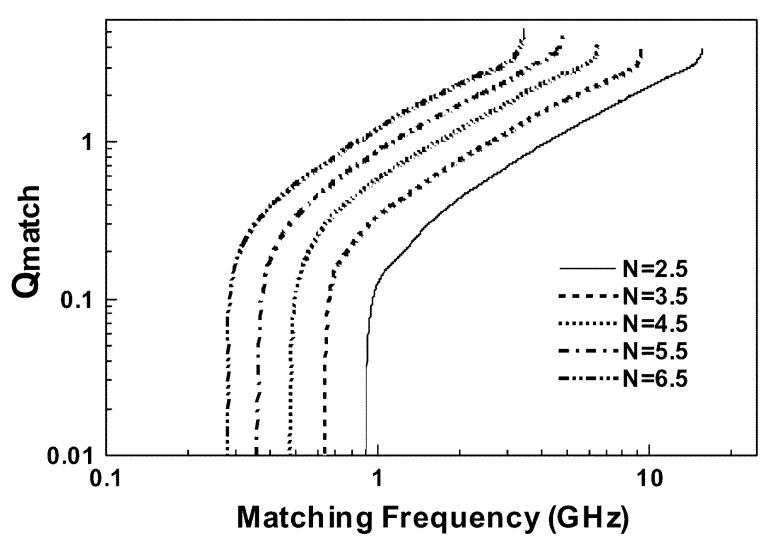

Fig. 8. Calculated $Q$ factors for high-impedance-type L-section matching circuits that use CMOS spiral inductors.

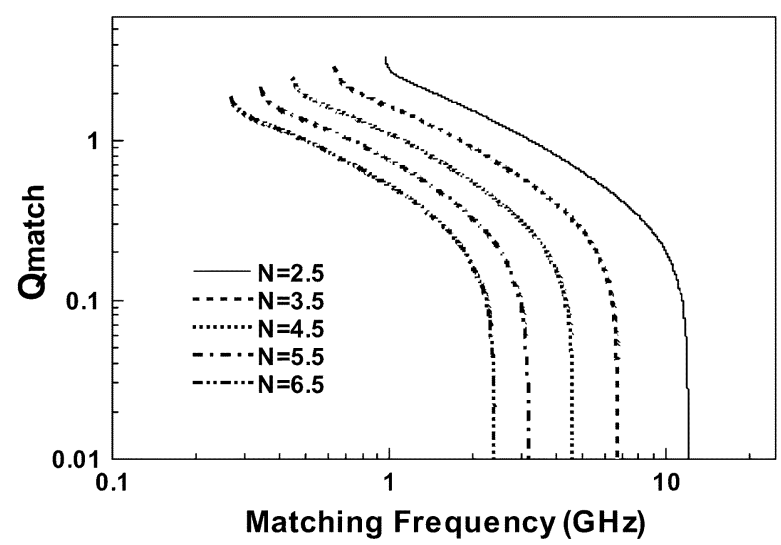

Fig. 9. Calculated $Q$ factors for low-impedance-type L-section matching circuits that use CMOS spiral inductors.

lossy matching circuit will be smaller due to the finite unloaded $Q$ factor $\left(Q_{\text {tank }}\right)$. The change ratio between $Q_{\text {match }}$ and $Q_{\mathrm{e}}$ is closely related to another parameter of interest, i.e., the load power ratio that accounts for the ratio of incident power dissipated in the load. From power conservation, the power dissipated in the load is equal to the incident power subtracting the power loss due to the spiral inductor, which can lead to the formulation of load power ratio, as shown in (24) at the bottom of this page.

Figs. 10 and 11 show the calculated results of load power ratio for the matching circuits shown in Fig. 7(a) and (b), respectively. For both types of matching circuits, one can see that the spiral inductor with a larger inductance or number of turns corresponds to a lower matching-frequency range. One can also compare Figs. 8 and 9 with Figs. 10 and 11 to conclude that a

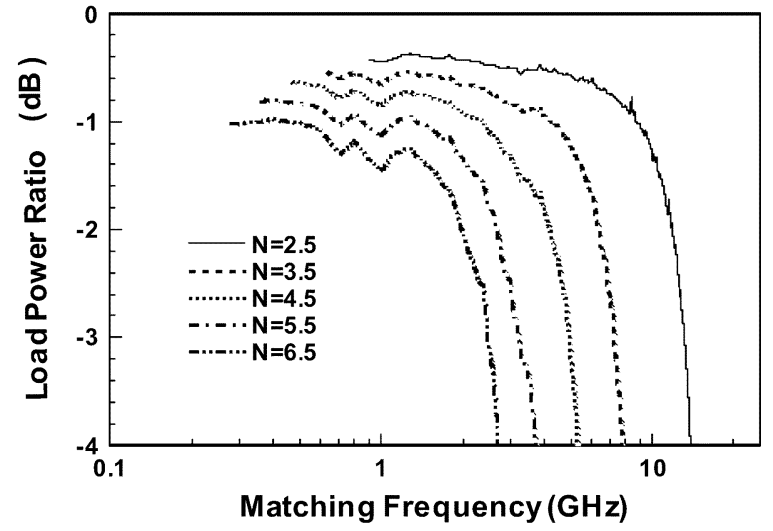

Fig. 10. Calculated load power ratios in decibels for high-impedance-type $\mathrm{L}$-section matching circuits that use CMOS spiral inductors.

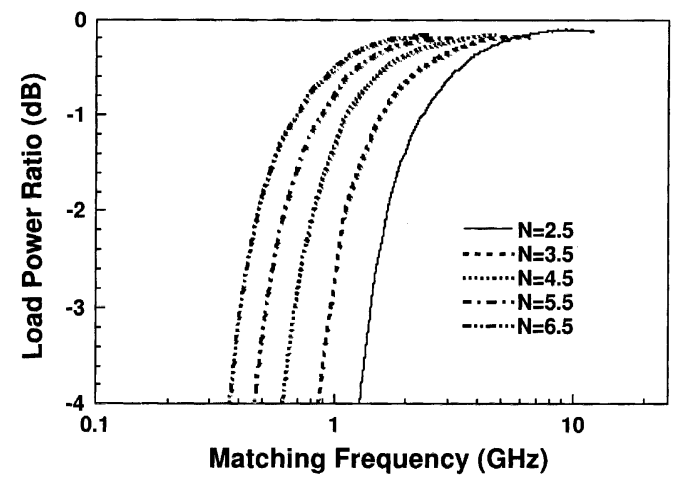

Fig. 11. Calculated load power ratios in decibels for low-impedance-type L-section matching circuits that use CMOS spiral inductors.

higher $Q$ factor for a higher impedance transform ratio is actually at the expense of lower load power ratio. In addition, the spiral inductor with a larger inductance or number of turns causes more loss to result in a lower load power ratio at the same higher $Q$-factor value.

\section{CONCLUSION}

A rigorous $S$-parameter formulation of the $Q$ factor for a spiral inductor in a generalized two-port configuration has been presented. The derivation employs a new complex-power concept and can reduce to the conventional $Q$-factor expression based on a one-port inductor model with the second port grounded. This $S$-parameter formulation has been also extended to predict the $Q$ factors for tank and matching circuits that use the spiral inductors quite successfully.

$$
\begin{aligned}
\text { Load Power Ratio } & =1-\frac{P_{l}^{\text {av }}}{P_{\text {inc }}^{\text {av }}} \\
& =1-\frac{\left[V^{+}\right]^{t}\left([U]-[S]^{t}[S]^{*}\right)\left[V^{+}\right]^{*}}{\left|V_{1}^{+}\right|^{2}\left|1+\Gamma_{\text {in }}\right|^{2}} \\
& =1-\frac{\left(1-\left|S_{11}\right|^{2}-\left|S_{21}\right|^{2}\right)+\left(1-\left|S_{22}\right|^{2}-\left|S_{12}\right|^{2}\right)|F|^{2}-2 \operatorname{Re}\left\{\left(S_{11} S_{12}^{*}+S_{21} S_{22}^{*}\right) F^{*}\right\}}{\left|1+S_{11}+S_{12} F\right|^{2}}
\end{aligned}
$$




\section{REFERENCES}

[1] C. P. Yue and S. S. Wong, "On-chip spiral inductors with patterned ground shields for Si-based RF IC's," IEEE J. Solid-State Circuits, vol. 33, pp. 743-752, May 1998.

[2] K. O, "Estimation methods for quality factors of inductors fabricated in silicon integrated circuit process technologies," IEEE J. Solid-State Circuits, vol. 33, pp. 1249-1252, Aug. 1998.

[3] H. Jiang, Y. Wang, J. A. Yeh, and N. C. Tien, "On-chip spiral inductors suspended over deep copper-lined cavities,' IEEE Trans. Microwave Theory Tech., vol. 48, pp. 2415-2423, Dec. 2000.

[4] Y. K. Koutsoyannopoulos and Y. Papananos, "Systematic analysis and modeling of integrated inductors and transformers in RF IC design," IEEE Trans. Circuits Syst. II, vol. 47, pp. 699-713, Aug. 2000.

[5] J. N. Burghartz, A. E. Ruehli, K. A. Jenkins, M. Soyuer, and D. N.-Ngoc, "Novel substrate contact structure for high- $Q$ silicon-integrated spiral inductors," in Int. Electron Devices Meeting Tech. Dig., 1997, pp. 3.2.1-3.2.4.

[6] Y. E. Chen, D. Heo, J. Laskar, and T. Anderson, "Investigation of $Q$ enhancement for inductors processed in BiCMOS technology," in Proc. IEEE RAWCON, 1999, pp. 263-266.

[7] L. F. Tiemeijer, D. Leenaerts, N. Pavlovic, and R. J. Havens, "Record $Q$ spiral inductors in standard CMOS," in Int. Electron Devices Meeting Tech. Dig., 2000, pp. 40.7.1-40.7.3.

[8] K. Naishadham and T. Durak, "Measurement-based closed-form modeling of surface-mounted RF components," IEEE Trans. Microwave Theory Tech., vol. 50, pp. 2276-2286, Oct. 2002.

[9] T. S. Horng, K. C. Peng, J. K. Jau, and Y. S. Tsai, " $S$-parameter formulation of quality factor for a spiral inductor in generalized two-port configuration," in IEEE MTT-S Int. Microwave Symp. Dig., 2003, pp. 255-258.

[10] C. Bowick, RF Circuit Design. Indianapolis, IN: H. W. Sams \& Company, 1982, ch. 4.

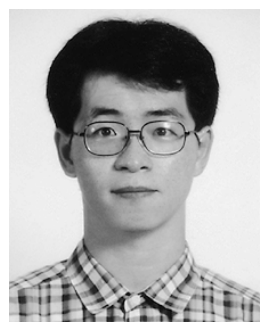

circuits and packages.
Tzyy-Sheng Horng (S'88-M'92) was born in Taichung, Taiwan, R.O.C., on December 7, 1963. He received the B.S.E.E. degree from the National Taiwan University, Taipei, Taiwan, R.O.C., in 1985, and the M.S.E.E. and Ph.D. degrees from the University of California at Los Angeles, in 1990 and 1992, respectively.

$\mathrm{He}$ is currently a Professor with the Department of Electrical Engineering, National Sun Yat-Sen University, Kaohsiung, Taiwan, R.O.C. His research interests are in the area of RF and microwave integrated

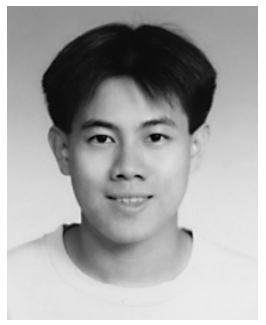

Kang-Chun Peng was born February 18, 1976, in Taipei, Taiwan, R.O.C. He received the B.S.E.E. and M.S.E.E. degrees from the National Sun Yat-Sen University, Kaohsiung, Taiwan, R.O.C., in 1998 and 2000, respectively, and is currently working toward the Ph.D. degree in electrical engineering at the National Sun Yat-Sen University.

His main research interests are microwave frequency synthesizers.

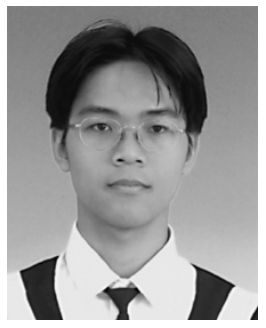

Je-Kuan Jau was born November 19, 1977, in Tainan, Taiwan, R.O.C. He received the B.S.E.E. degree from the National Sun Yat-Sen University, Kaohsiung, Taiwan, R.O.C., in 1999, and is currently working toward the Ph.D. degree in electrical engineering at the National Sun Yat-Sen University.

His main research interests are high-efficiency power amplifiers.

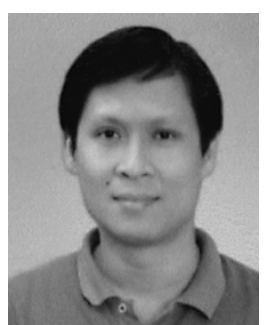

Yu-Shun Tsai was born March 3, 1963, in Kaohsiung, Taiwan, R.O.C. He received the M.S.E.E. degree from the National Chiao Tung University, Hsinchu, Taiwan, R.O.C., in 1991, and is currently working toward the Ph.D. degree in electrical engineering at the National Sun Yat-Sen University, Kaohsiung, Taiwan, R.O.C.

His research interests include design and modeling of microwave components. 